\title{
Combined Endoscopic Therapy And Percutaneous Approach in Patients with Biliary Strictures After Adult Right-Lobe Living-Donor Liver Transplantation
}

\author{
Horng-Ren Yang ${ }^{1}$, Ashok Thorat ${ }^{1}$, Wen-Hsin Huang ${ }^{2}$, Cheng-Ju Yu ${ }^{2}$, Te-Hung Chen ${ }^{1}$, Shih-Chao Hsu ${ }^{1}$, \\ Kin-Shing Poon ${ }^{3}$, Long-Bin Jeng ${ }^{1}$
}

\author{
Corresponding author: \\ Long-Bin Jeng, MD \\ Organ Transplantation Center \\ China Medical University Hospital \\ 2, Yuh-Der Road, Taichung, Taiwan \\ 40447 \\ Tel : 04-22052121Ext.1765 \\ Fax : 04-22029083 \\ E-mail: liver.cmuh@gmail.com
}

Received: 05.05.2019

Accepted: 10.06.2019

Copyright (1) Celsius Publishing House www.sgo-iasgo.com
${ }^{1}$ Organ transplantation centre, China Medical University Hospital, Taichung, Taiwan ${ }^{2}$ Digestive Medicine, Department of Internal Medicine, China Medical University Hospital, Taichung, Taiwan

${ }^{3}$ Department of Anaesthesiology, China Medical University Hospital, Taichung, Taiwan

\section{ABSTRACT}

Background: Endoscopic retrograde cholangiography (ERC) with balloon dilation and stenting is the curative treatment for biliary anastomotic (AS) strictures after living donor liver transplantation (LDLT). The aim of this study was to assess the technical feasibility and clinical outcomes of combined endoscopic and percutaneous therapy for biliary AS stricture in adult right-lobe living donor liver transplantation with duct to duct anastomosis.

Materials and methods: Between January 2008 and December 2015, 648 patients receiving right-lobe LDLT in our hospital were analyzed for biliary complications. When biliary strictures were diagnosed, patients underwent ERC first as a diagnostic as well as treatment modality to dilate the strictures and biliary stenting. If ERC failed, percutaneous transhepatic cholangiography (PTC) and/or combined PTC and ERC were done.

Results: Eighty-two biliary strictures were diagnosed in 648 recipients (13\%; 82/648) at an average of 256 (7-1979) days after LDLT. Among 82 patients (age $52.6 \pm 9.6$ years, range 24-72), 40 patients were successfully treated by ERC, 5 by PTC, and 9 by combined PTC and ERC. In successful ERC with an average of $5.03 \pm 2.96$ sessions, $11 \pm 11.0$ plastic stents in total and $2 \pm 1$ stents at each ERC session were placed. The mean time to stricture resolution was $708 \pm 532$ (28-1967), $298 \pm 135$ (145-398), and $499 \pm 239$ (288-827) days for patients receiving ERC, PTC, and combined PTC and ERC, respectively.

Conclusions: Biliary complications following LDLT is associated with high mortality. In severe biliary strictures, the procedure that combines PTC and ERC can be curative in patients with prior failed ERC treatment.

Key words: biliary strictures, percutaneous transhepatic cholangiography, endoscopic retrograde cholangiography, anastomotic and non-anastomotic biliary strictures, living donor liver transplantation

\section{INTRODUCTION}

Biliary strictures are most common delayed biliary complications occurring after liver transplantation (LT) that were reported to occur up-to $35 \%$ of LT recipients $(1,2)$. However, with the advent of improved surgical techniques and 
increased experience in LT, the overall incidence of anastomotic strictures is reduced, occurring in approximately $13 \%$ of the recipients. Nevertheless, biliary stricture remains an important cause of morbidity after LT and in severe cases can lead to graft loss. Smaller caliber of the hepatic ducts in partial grafts in LDLT makes them prone for biliary strictures more often compared to the deceased donor liver transplantation (DDLT) recipients. In LDLT, there is often a risk of devascularization of the bile duct during the hilar dissection of the donor as well as the potential risk of bile leak from the cut surface of liver allograft, causing fibrotic changes around the biliary anastomosis (3). The etiology of post-LT biliary complications is multifactorial and includes arterial, technical (single or multiple anastomoses) as well as immunologic components that determine the type and location of biliary strictures and their clinical presentation. However, the strictures in most cases remain undetected until hepatic dysfunction or infection becomes apparent. Biliary strictures are classified as AS and non-anastomotic (NAS) depending upon the site of occurrence. AS biliary strictures are mainly secondary to surgical technique of biliary reconstruction whereas NAS strictures are primarily due to hepatic arterial complications or from complex immunological factors (4).

Surgical treatment such as re-exploration and revision of anastomosis or Roux-en $Y$ has been the traditional approach in treating severe biliary strictures. However, advances in therapeutic and diagnostic endoscopic procedures have made the nonoperative management of biliary complications as a preferred modality for treating the biliary strictures that develop after LT. In recent era, the ERC with balloon dilation (BD) and placement of increasing numbers of plastic stents has become the principal modality of treatment for AS biliary strictures after LT. The PTC and surgical management are generally reserved for failures of endoscopic therapy or for anatomic variants that are not suitable for ERC. The response to treatment depends upon the timing of its occurrence, type and severity of stricture. AS biliary strictures that occur early after LT (less than one month) is related to pericholedochal edema and easily resolves after ERC and $B D$ with short stent duration (four to six months) compared to late occurring AS biliary strictures that require biliary stent for longer duration $(5,6)$.

At present, the preferred endoscopic approach for AS stricture is repeated aggressive dilation of the stenosed bile duct and, if feasible, insertion of multiple plastic stents. In patients with severe strictures or with NAS strictures, endoscopic biliary access is technically difficult. In such patients a combined endoscopic and percutaneous approach proves quite useful. The aim of this study was to assess the technical feasibility and clinical outcomes of combined endoscopic and percutaneous therapy for AS biliary stricture in adult right-lobe LDLT with duct to duct anastomosis.

\section{MATERIALS AND METHODS}

Between January 2008 and December 2015, 648 patients receiving right-lobe living donor liver transplantation at China Medical University Hospital, Taiwan were analyzed for biliary complications with minimum of two years of follow up.The patients that developed biliary strictures requiring some form of intervention such as ERC with BD and stenting and/or PTC were included in present study and the outcome of the treatment modalities, graft function and long-term survival were retrospectively studied. The patients that underwent a successful intervention therapy were further sub-grouped into group A (patients treated with ERC) and group B (patients treated by PTC and PTC+ERC). The characteristics of biliary strictures in each group and the outcome after the therapeutic procedures to treat biliary structures were analyzed and compared.

The demographic data, medical records and pretransplant laboratory data of the recipients were assessed for any abnormality. The complications directly related to hepatic artery (HA) were also recorded and retrospectively analyzed in patients with biliary complications. The donors were evaluated by standard donor survey protocol. The evaluation of donor liver was done by computed tomography (CT) scan and magnetic resonance imaging (MRI) along with the 3-D reconstruction of CT liver images and the liver segmental anatomy, the approximate volume of both the liver lobes and biliary tract anatomy was studied in detail (7).

The biliary reconstruction was duct-to-duct in all the cases. After arterial anastomosis was completed, recipient bile duct was assessed, and the adequate length of biliary duct was mobilized to have a tension free anastomosis. The posterior layer of biliary anastomosis was sutured in continuous fashion whereas anterior layer was completed by interrupted 5-0 prolene sutures.We do not place biliary drain intraoperatively and cholangiography in postoperative period is done only if clinically indicated. Immunosuppressive therapy was as per the institution protocol described earlier (8). This retrospective study was approved by institutional review board. 


\section{Initial diagnosis of biliary stricture and choice of treatment modality}

Patients were followed up by protocol ultrasound (US) of abdomen after LDLT (7). Any patients with postoperative rise in bilirubin were further assessed by following laboratory tests:serum level of alkaline phosphatase, gamma glutamyl transferase, total and direct bilirubin, and alanine aminotransferase. Diagnosis of biliary strictures was based on radiological imaging studies in the background of raised serum bilirubin. US abdomen with Doppler study of the liver was the initial modality of investigation that was followed by magnetic resonance cholangiopancreatography (MRCP) when biliary stricture was suspected. The timing of occurrence of biliary strictures from the date of LDLT, the site of occurrence (anastomotic or non-anastomotic), morphology (pouched or nonpouched, branched or unbranched) and intrahepatic biliary radical dilatation were noted. An AS stricture was defined as a focal narrowing at the site of the biliary anastomosis whereas 1 or more focal areas of narrowing of the bile ducts proximal to a biliary anastomosis was considered as NAS biliary stricture. Length and diameter of stricture were measured. Endoscopic intervention was first line of therapy in all biliary stricture patients. Figure 1 shows the protocol of the treatment algorithm for post LDLT biliary strictures and different modalities of treatment.

\section{Endoscopic protocol}

When diagnosis of biliary stricture was confirmed by radiological imaging tests, ERC was used as firstline modality for the management that consisted of passing the stricture with a guide wire, $B D$, and placement of one or more 7-10 Fr plastic stents depending upon the location and severity of the stricture. After the guidewire (0.025 or 0.035 inch) was passed through the duct across the stricture, endoscopic sphincterotomy or endoscopic papillary BD was performed to allow placement of stents (fig. 2 b, c). Figure 2, 3, 4, and 5 shows different types of strictures in relation to morphology and site with successful endoscopic procedures and insertion of single or multiple stents. If ERC failed, stricture dilatation was accomplished by a combined PTC and ERC approach (Rendezeus Technique). Figure 6 shows the images of combined approach of ERC and PTC. Failure of ERC procedure was defined as inability to pass guidewire proximal to the stricture that needed combinationPTC. Endoscopic session was repeated every 3 months for evaluation of the stricture and replacement of stent. Clinical success of interventional procedures was defined as reduction of biliary stasis and normalization of the liver enzymes or a trend towards normalization. Resolution of stricture was further confirmed by follow up ballon occlusion cholangiography (fig. $2 d$ ). Recurrence of biliary strictures was defined as

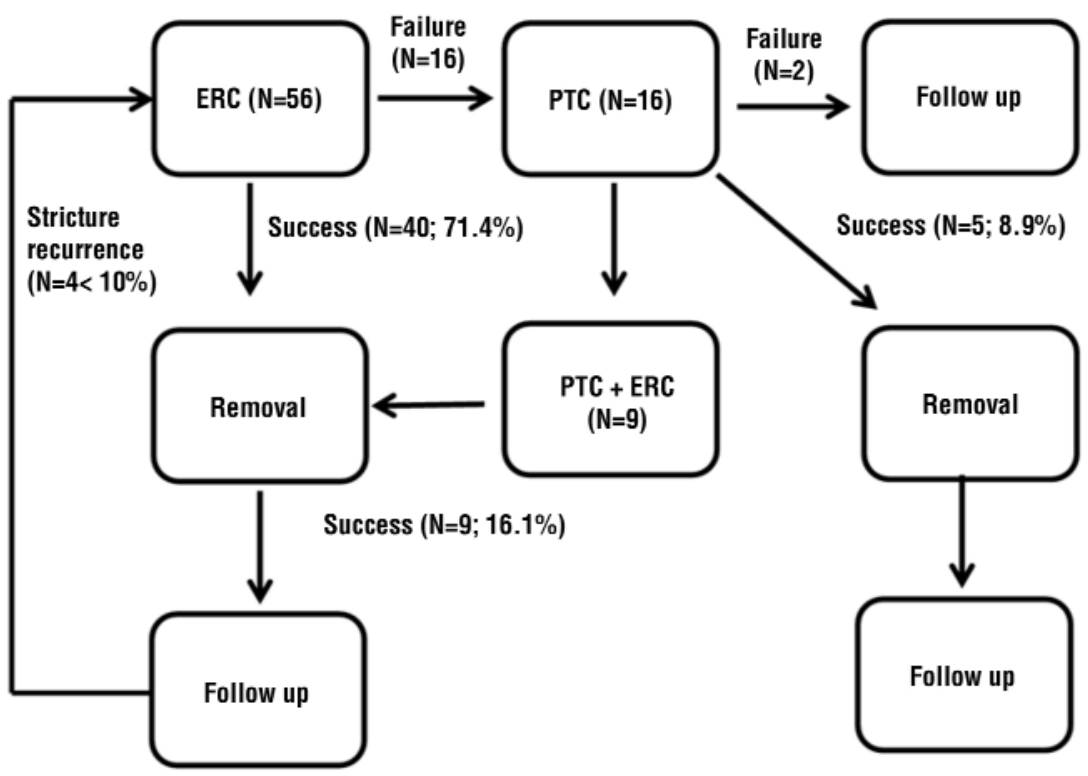

Figure 1 - Treatment algorithm of biliary stricture after LDLT 
Figure 2 - Fork shaped AS stricture and successful management by ERCP. a) Fork-shaped stricture. b) Endoscopic BD.

c) Stents placement. d) Balloon occlusion cholangiography showed resolution of anastomotic stricture.
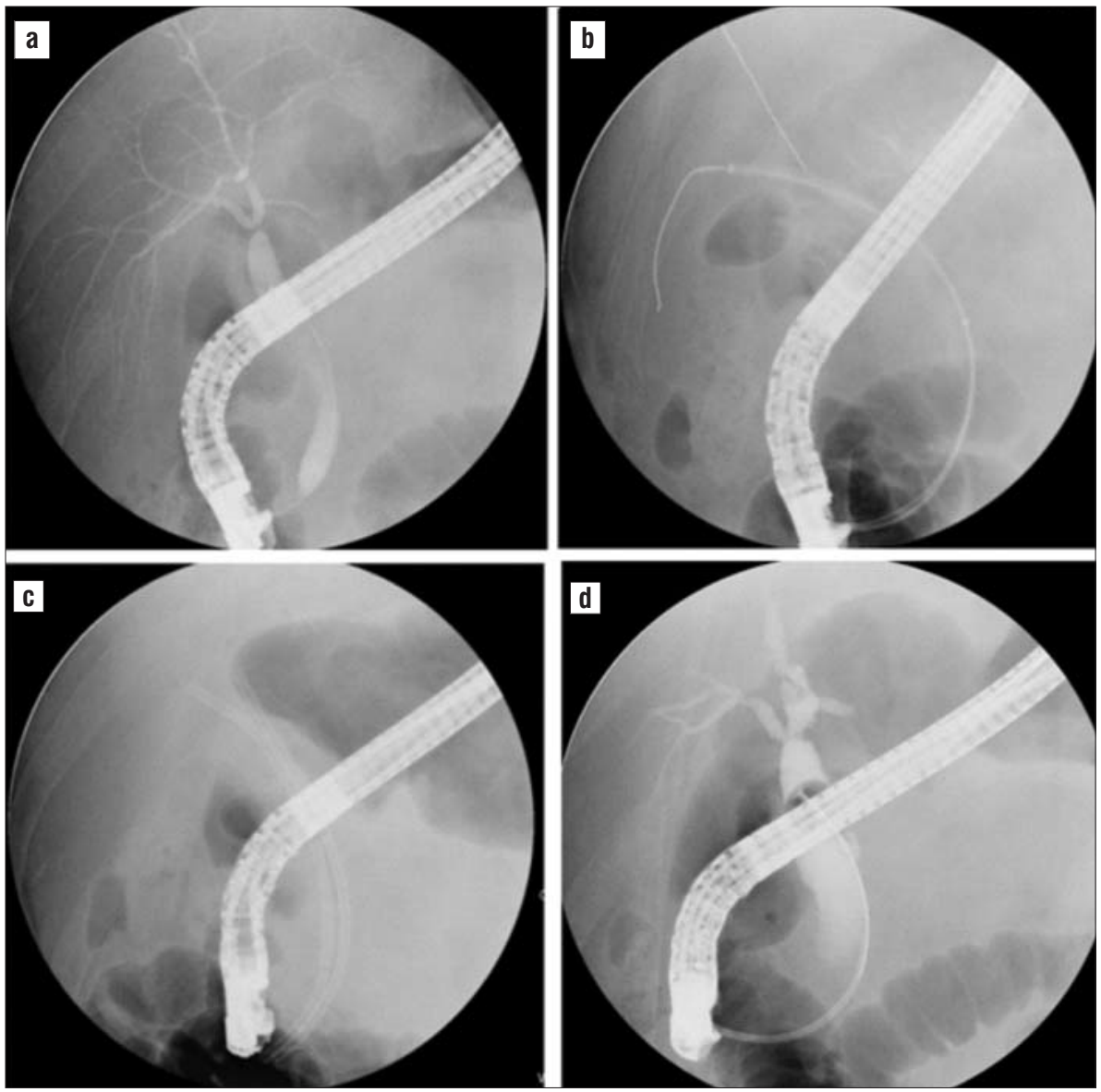

recrudescence of biliary stricture signs and Group comparisons were done utilizing 2-tailed symptoms after initial clinical success.

\section{Statistical analysis}

The data for the present retros-pective study were analyzed with PASW statistics v18.0 (SPSS Inc.). t-test for continuous variables and $\chi^{2}$ test for categorical variables. The risk factor analysis was performed using univariate analyses. Results were presented as mean values \pm standard deviation (SD). All tests were two-sided and $p<0.05$ was considered as statistically significant.
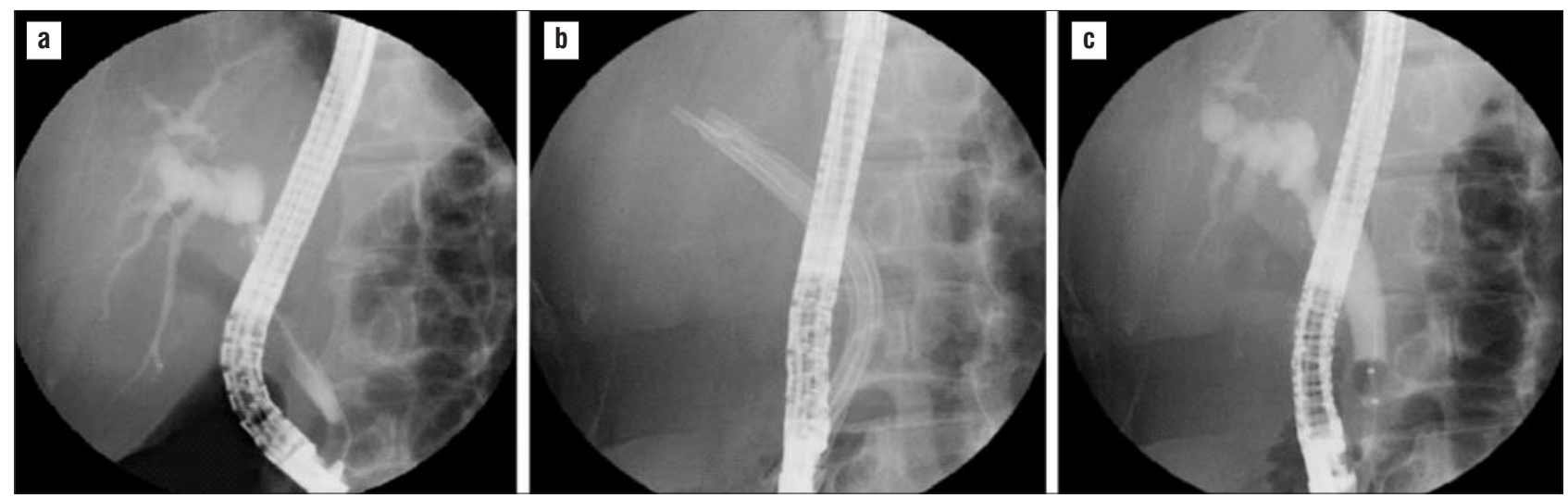

Figure 3 - Unbranched AS stricture management. a) Unbranched stricture morphology. b) Placement of 5 plastic stents.

c) Cholangiography showing stricture resolution. 
Figure 4 - Multi-branched AS stricture management. a) Strictureanatomy. b) Endoscopic BD.

c) Placement of stents.

d) Stricture resolution on follow up cholangiography.
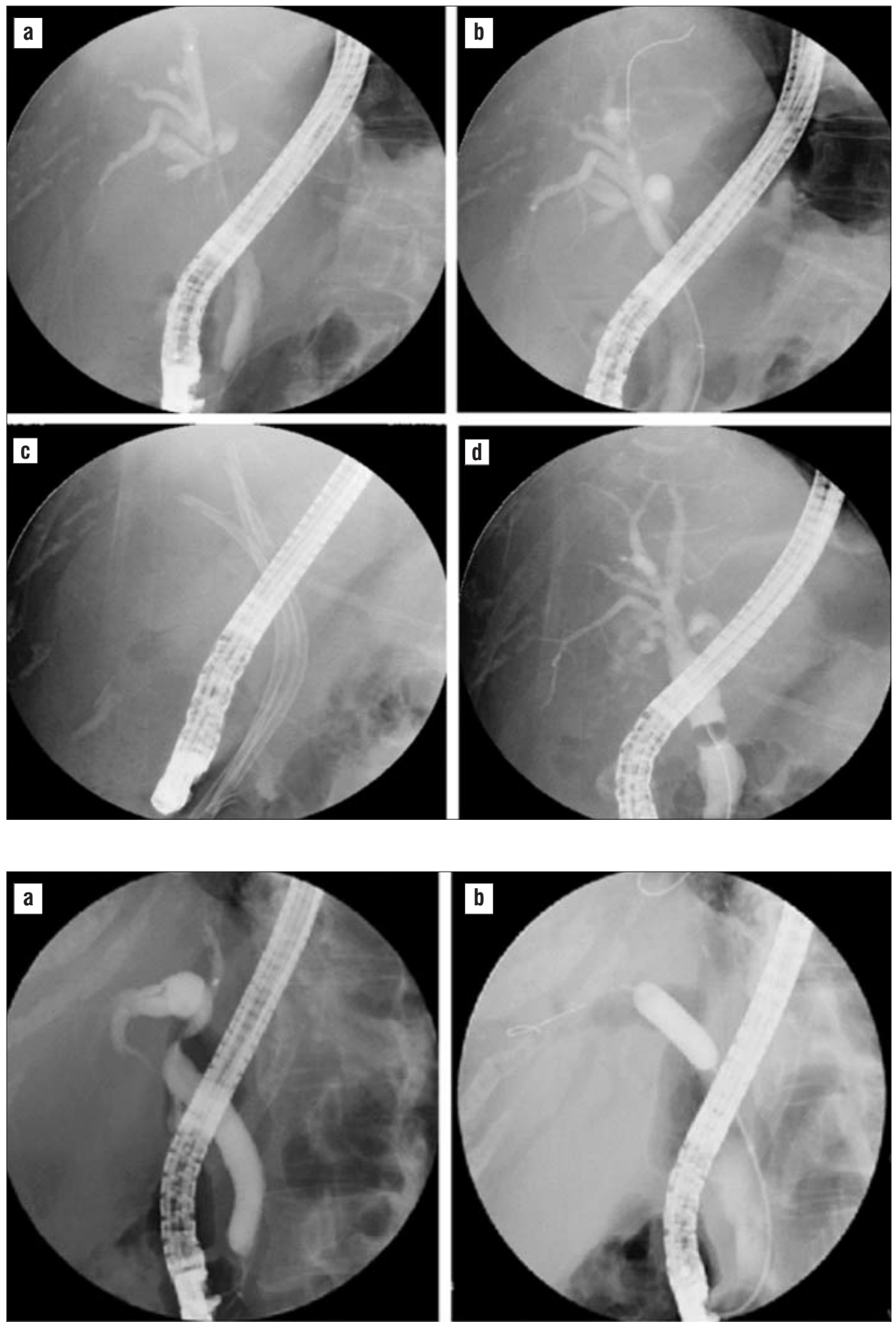

Figure 5 - Unbranched AS stricture. a) Strictureanatomy. b) Endoscopic BD. c) Placement of 8 stents. d) Cholangiography showing Stricture resolution.
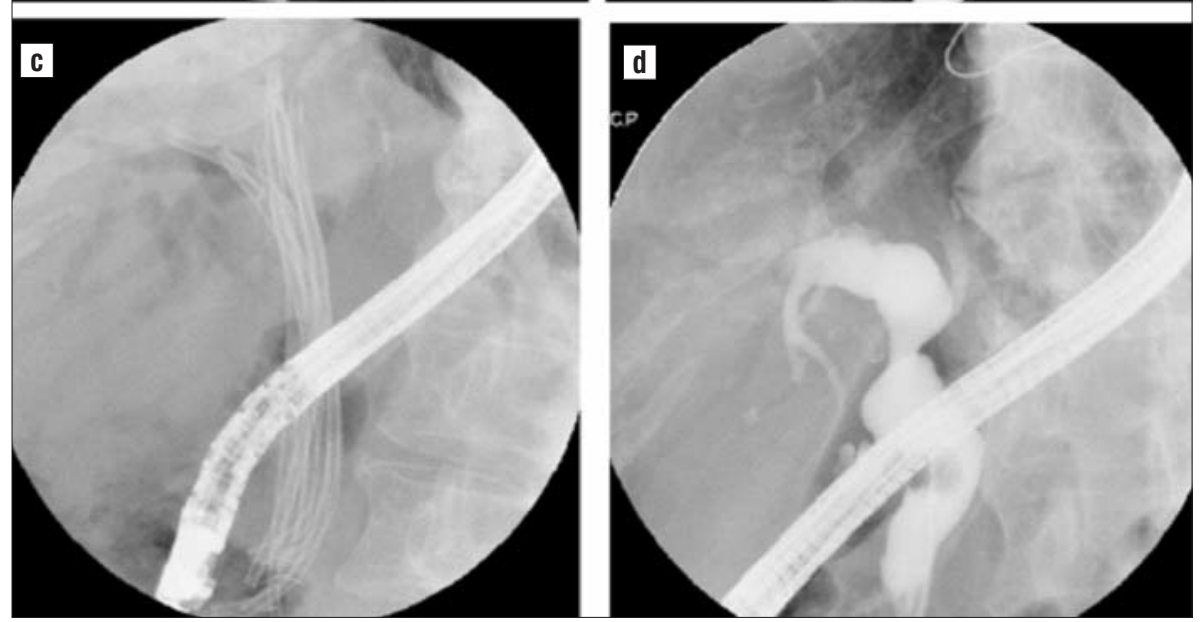

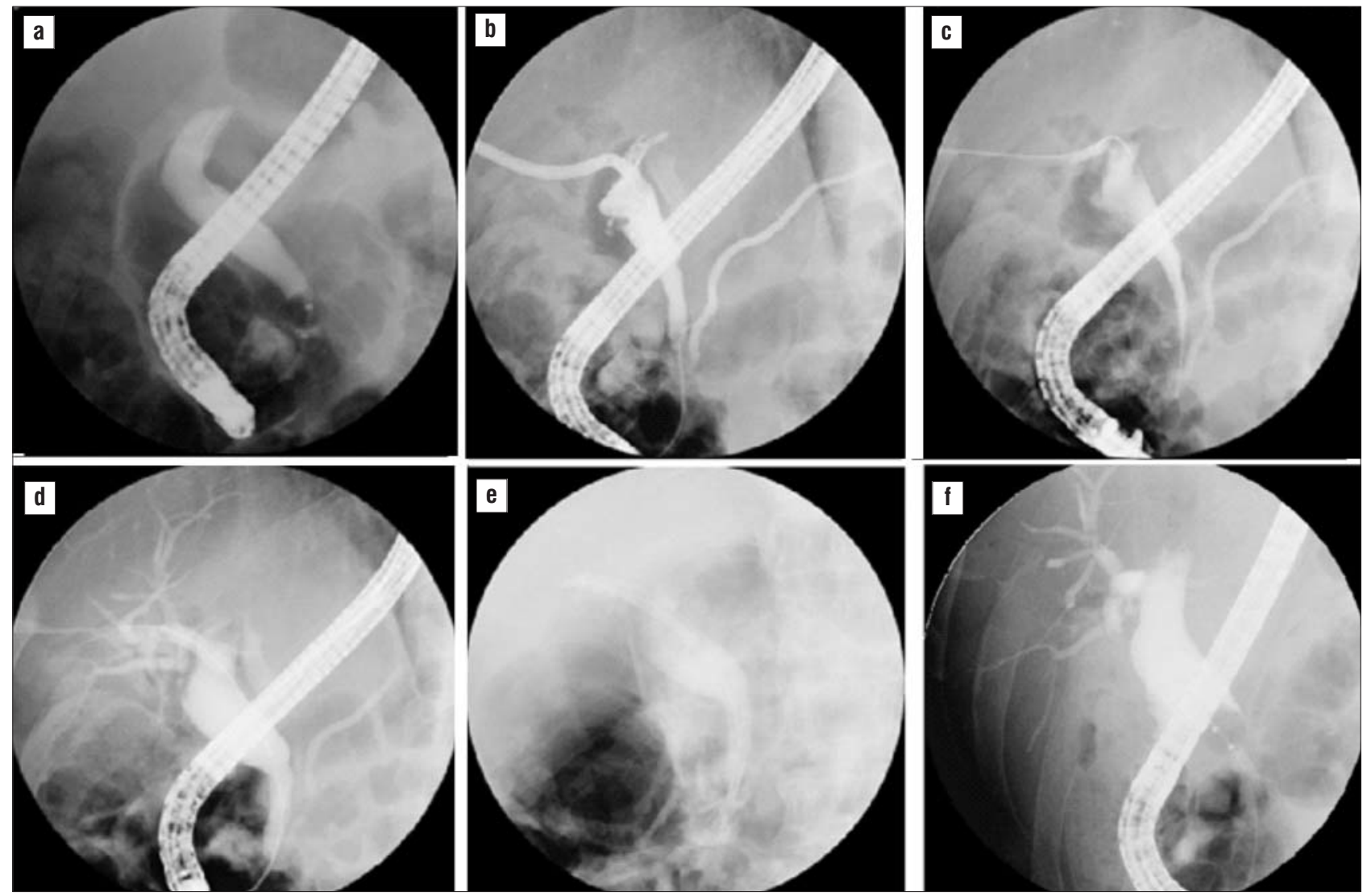

Figure 6 - ERC combined with PTC (Rendezeus technique) for the management of NAS biliary stricture.

a) Failed ERC at pouched AS biliary stricture. b) PTC. c) Rendezvous technique (PTC $>$ ERC).

d) Endoscopic balloon dilation. e) Stent placement. f) complete resolution of biliary stricture.

\section{RESULTS}

All the studied recipients received right lobe graft. The biliary tract was primarily reconstructed by a ductto-duct anastomosis in all the patients. Eighty-two recipients with a mean age $52.6 \pm 9.6(24-72)$ years (Male: Female, 59:23) were diagnosed to have biliary strictures among the 648 LDLT recipients (12.65\%; $82 / 648)$ at an average of 256 (7-1979) days after LDLT. The primary indications for LDLT are shown in table 1. Biliary reconstruction was done by standard duct-to duct anastomotic technique in all the patients. Ten patients developed additional complication of bile leak along with biliary stricture. The AS strictures were diagnosed in 76 patients (93\%; 76/82) whereas only 6 patients had NAS biliary strictures at intrahepatic location (table 2). Ten patients had bile leak diagnosed sometime after transplantation due to bile content in abdominal drain, however, the site of bile leak couldn't be confirmed.

Forty patients $(n=40)$ were successfully treated by ERC alone and 14 patients required PTC. Among the PTC treated patients 9 patients received some form of
Table 1 - Basic characteristics of patients with biliary stricture after LDLT

\begin{tabular}{lc}
\hline Basic characteristics & No. patients (N=82) \\
\hline Age (year) & $52.6 \pm 9.6(24-72)$ \\
\hline Gender & $59(72 \%)$ \\
Male & $23(28 \%)$ \\
Female & \\
\hline Indications for LDLT & $33(40 \%)$ \\
HCC & $15(18 \%)$ \\
HBV/ESLD & $11(13 \%)$ \\
Alcohol/ESLD & $7(9 \%)$ \\
HCV/ESLD & $5(6 \%)$ \\
HBV/AE & $3(4 \%)$ \\
Autoimmune/ESLD & $2(2 \%)$ \\
HBV+HCV/ESLD & $6(8 \%)$ \\
Other & \\
\hdashline Biliary complication & $72(88 \%)$ \\
Stricture only & $10(12 \%)$ \\
Stricture + Leakage & \\
\hline Stricture site & $76(93 \%)$ \\
Anastomosis & $6(7 \%)$ \\
Non-anastomosis & $256 \pm 372(7-1979)$ \\
\hline Time to Stricture (day) &
\end{tabular}


Table 2 - Results of patients with biliary strictures after LDLT receiving ERC and PTC treatment

\begin{tabular}{|c|c|c|c|}
\hline & $\begin{array}{c}\text { Group A } \\
(n=40)\end{array}$ & $\begin{array}{c}\text { Group B } \\
(n=14)\end{array}$ & $P$ value \\
\hline Age (years) & $51.4 \pm 9.9(20-72)$ & $51.86 \pm 10.2(24-65)$ & 0.8834 \\
\hline $\begin{array}{l}\text { Gender } \\
\text { Female } \\
\text { Male }\end{array}$ & $\begin{array}{l}12(30 \%) \\
28(70 \%)\end{array}$ & $\begin{array}{l}6(42.9 \%) \\
8(57.1 \%)\end{array}$ & 0.3798 \\
\hline $\begin{array}{l}\text { Biliary complication } \\
\text { Stricture only } \\
\text { Stricture } \pm \text { Leakage }\end{array}$ & $\begin{array}{c}35(87.5 \%) \\
5(12.5 \%)\end{array}$ & $\begin{array}{c}11(78.6 \%) \\
3(21.4 \%)\end{array}$ & 0.4183 \\
\hline $\begin{array}{l}\text { Stricture site } \\
\text { Anastomosis } \\
\text { Non-anastomosis }\end{array}$ & $\begin{array}{c}38(95 \%) \\
2(5 \%)\end{array}$ & $\begin{array}{c}13(92.9 \%) \\
1(7.1 \%)\end{array}$ & 0.7632 \\
\hline Pouched & $8(20 \%)$ & $6(66.7 \%)$ & 0.0051 \\
\hline Non-pouched & $32(80 \%)$ & $3(33.3 \%)$ & \\
\hline Branched & $27(67.5 \%)$ & $6(66.7 \%)$ & 0.9616 \\
\hline Non-branched & $13(32.5 \%)$ & $3(33.3 \%)$ & \\
\hline Time to stricture & $288 \pm 422(7-1979)$ & $225 \pm 255(11-826)$ & 0.6023 \\
\hline Total stents & $11.5 \pm 11.0(1-64)$ & $8.8 \pm 6.0(1-19)$ & 0.4843 \\
\hline Average stents & $2.05 \pm 0.71(1-4.6)$ & $2.23 \pm 0.64(1-2.8)$ & 0.4895 \\
\hline ERCP No. & $5.03 \pm 2.96(1-13)$ & $3.78 \pm 2.68(2-14)$ & 0.2515 \\
\hline Time of resolution & $706 \pm 526(28-1967)$ & $1074 \pm 612(145-827)$ & 0.0352 \\
\hline Stricture recurrence & $4(10 \%)$ & $0(0 \%)$ & \\
\hline Time of follow up & $970 \pm 643(70-2228)$ & $1138 \pm 664(233-2397)$ & 0.4067 \\
\hline
\end{tabular}

Group A: ERC; Group B: PTC and PTC+ERC

ERC treatment as well. Treatment failed in two patients whereas six patients are still under treatment. The remaining twenty patients (24\%; 20/ 82) died during the duration of treatment. Five patients among them died due to biliary sepsis.

\section{ERC vs Combined approach $(E R C+P T C)$}

The patients that were successfully treated either by endoscopic and/or percutaneous therapeutic modalities $(n=54)$ were further divided into two subgroups and factors related to the strictures and their response to treatment were assessed. Group A patients comprised of 40 patients (Male:Female, 28:12) that underwent successful endoscopic treatment with an average of $5 \pm 3$ sessions of ERC whereas 14 group B patients (Male: Female, 8:6) underwent combined ERC and PTC with average $3 \pm 2$ sessions. Each ERC session of group $A$ and $B$ patients involved average $2 \pm 1$ stent insertion each $(p=0.48)$. The mean time to stricture resolution was 708 \pm 532 (281967) and $1074 \pm 612$ (145-827) days for patients receiving ERC and combined PTC/ERC, respectively $(p=0.03)$. Majority of strictures were AS in nature in all studied patients (95\% in group A and $92.9 \%$ in group B). Morphology of the stricture was an important determinant factor, non-pouched being more common in group A (80\%) whereas pouched strictures were common in group B patients $(66.7 \%)$. Pouched shape stricture was the only factor associated with failed ERC $(P<0.05)$ (fig. 6).

During a follow-up period of $976 \pm 643$ days, four patients receiving ERC developed recurrent strictures whereas there were no recurrent strictures for group B. The four recurrent strictures were successfully treated by repeated ERC. However, the duration of the treatment was longer for patients in group $B$.

In all patients, the hepatic artery was patent at the time of diagnosis as determined by Doppler US evaluation of the abdomen.

\section{$A S$ and NAS biliary strictures}

Among the 54 successfully treated patients, 51 patients had AS strictures whereas only three patients had NAS strictures (table 3$) .68 \%(n=35)$ patients with AS strictures underwent successful treatment by ERCP and stenting whereas 16 patients required ERC and/or PTC to manage the strictures. The incidence of NAS strictures was very low and majority of them $(2 / 3)$ required ERC combined with PTC approach to achieve 
Table 3 - Outcome in patients with anastomotic and non-anastomotic strictures

\begin{tabular}{lccc}
\hline & $\begin{array}{c}\text { Group } \mathbf{A} \\
(\mathbf{n}=\mathbf{5 1})\end{array}$ & $\begin{array}{c}\text { Group } \mathbf{B} \\
(\mathbf{n}=\mathbf{3})\end{array}$ & P value \\
\hline Age (years) & $51.6 \pm 10(24-72)$ & $49 \pm 4.5(43-53)$ & 0.3489 \\
$\begin{array}{l}\text { Gender } \\
\text { Female }\end{array}$ & $18(35.3 \%)$ & 0 & 0.1075 \\
$\quad$ Male & $33(74.9 \%)$ & $3(100 \%)$ & \\
Time to stricture & $278 \pm 279(7-1979)$ & $148 \pm 135.6(21-336)$ & 0.2857 \\
\hdashline Time of resolution & $791 \pm 564(45-2504)$ & $1460 \pm 296(1080-1803)$ & 0.0253 \\
\hdashline Treatment & $35(68.6 \%)$ & $1(33.3 \%)$ & \\
$\quad$ ERC & $6(11.8 \%)$ & $2(66.7 \%)$ & \\
PTC & $10(19.6 \%)$ & 0 & \\
ERC+PTC & & & \\
\hline
\end{tabular}

* Group A : Anastomotic stricture; Group B : Non-anastomotic stricture

stricture resolution. AS strictures showed early resolution as compared to NAS strictures $(791+564$ days vs $1460+296$ days, $\mathrm{p}=0.02$ ). However, time to stricture development was faster in NAS strictures. AS strictures showed different morphological types such as fork shaped, branched (single or multi-branched) and unbranched requiring multiple stent placements (fig. 2, 3, 4, 5) whereas non-pouched morphological variant was common in NAS-strictures that required combined technique for treatment.

\section{DISCUSSION}

Even after technical advances and standardization of effective techniques for biliary reconstruction, the biliary stricture remains a major biliary complication after LT and reported to occur in substantial population of the LDLT recipients that affects the long-term outcome as well as graft survival $(9,10,11)$. In the metaanalysis of the selected studies, Aparicio et al stated an increased absolute risk for biliary strictures for LDLT patients ( $35 \%$ vs $13 \%, 95 \% \mathrm{Cl},-0.24$ to $-0.08 ; p=0.0001$ ) (6). In this study, the biliary strictures occurred in $12.65 \%$ of the patients which is lower than the reported standard biliary complication rates. This can be attributed to the refined biliary reconstruction technique (described earlier) and a very low incidence of hepatic arterial complications. All the strictures were treated endoscopically, and none required operative intervention. ERC was the first-line treatment modality for post-LDLT anastomotic biliary strictures in our series. In patients with failed ERC due to severe stricture and/or intrahepatic NAS biliary strictures, the therapy wascombined with PTC. Thus, a combined approach of ERC and PTC proved to be $96.4 \%$ successful in 54 patients with no recurrence of the stricture till the latest follow up. However, 4 patients that underwent only ERC and stenting had recurrence of the AS strictures which were successfully treated by repeated ERC.

The AS biliary stricture is a focal narrowing around the biliary anastomosis secondary to fibrotic healing and remains most common type of stricture and the majority occur within the first year after transplant (13). Post-LDLT AS biliary strictures that appear early in postoperative period are usually technical issues such as smaller caliber of the bile ducts, inappropriate suture material, tension at the anastomosis and/or improper surgical technique (14). The devascularization of the bile duct at the hilar dissection during donor hepatectomy in LDLT is an important predisposing factor for strictures. Bile leak that develops in immediate post-LDLT period has been considered as an important risk factor for the development of AS smaller. The bile leak around the anastomosis can cause local inflammation, edema and fibrosis, thus, causing formation of AS strictures $(2,15)$. Other risk factors for biliary strictures include bile duct diameter, a graft with multiple bile ducts, increased graft cold ischemia time, hepatic artery stenosis, and acute cellular rejection $(16,17)$. In present study cohort, the prevalence of AS strictures was $11.7 \%$ (76/648) whereas the NAS strictures were diagnosed in $<1 \%$ of the patients constituting $7 \%$ of biliary stricture complications which compares well with previously published literature (4-10\% NAS strictures in recent studies) $(1,2,18)$.

Patients with biliary strictures are usually asymptomatic and presents with hyperbilirubinemia with elevated liver enzymes. Acute rejection or other vascular etiologies must be excluded before considering the biliary stricture diagnosis. A high index of suspicion 
must be maintained in patients with serum bilirubin levels $>1.5 \mathrm{mg} / \mathrm{dl}$ (19). Mildly elevated liver enzymes with adequate trough levels of tacrolimus virtually eliminates acute cellular rejection as differential diagnosis. If doubt exists, liver biopsy can be performed. In our study, after excluding the acute rejection, US abdomen with doppler evaluation was the initial imaging study to exclude the vascular cause that was followed by MRCP. The sensitivity and specificity of MRCP in establishing the diagnosis of biliary strictures is close to $90 \%$ and, thus, it helps avoiding unnecessary endoscopic intervention in absence of direct evidence of biliary strictures. However, the chief disadvantage of MRCP is the lack of its therapeutic ability.

In recent era, advances in endoscopic therapy and interventional radiology have improved the outcome of post-LDLT biliary strictures by decreasing the need for surgical repair that has high morbidity. ERC has become the first line of treatment for biliary strictures in patients with duct-to-duct biliary anastomosis at our center which is considered as gold standard for establishing the diagnosis as well as therapeutic intervention in the same setting (20). All the patients in this study underwent ERC with BD followed by insertion of plastic stents. Twenty patients (24.3\%) with stricture died and/or lost follow up before resolution of the stricture. Five of these patients died secondary to sepsis after the ERC and PTC procedures. We strongly recommend application of aggressive endoscopic therapy for biliary AS stricture that can be combined with PTC if stricture can not be traversed by ERC guided stents. In this study cohort, we performed ERC with BD followed by placement of progressively greater numbers of stents across the strictures. This achieved a sustained patency in $90 \%$ (36/40) of the patients treated by ERC and stenting. Four patients developed recurrence and were successfully treated by repeated ERC procedures. Multiple, side-by-side biliary stents have shown better results than single stents in patients with biliary AS strictures after $\operatorname{LDLT}(21,22)$. Every three months ERC with BD sessions were repeated and progressively increased diameter plastic stents of $7 \mathrm{Fr}$ to $10 \mathrm{Fr}$ were inserted until the strictures were resolved. In group $A$ subgroup patients average ERC sessions were $5 \pm 3$ (1-13) and two or more stents were inserted. In small subgroup of patients, the early AS biliary stricture within 2 months post-LDLT can be a transient narrowing that occurs due to postoperative periductal edema and inflammation. Such patients respond well to ERC and $\mathrm{BD}$ alone or may need temporary stenting for short duration as compared to severe strictures (23). The long-term success rate of ERC was $74 \%$ for the AS in our series as described in standard studies worldwide $(1,21,22,24)$.

When the endoscopic treatment failed, PTC was performed as a rescue procedure. The morphology of biliary stricture,especially pouched AS stricture, was the determining factor associated with failed ERC $(P<0.05)$ in this study. In subset of LDLT recipients, morphological deformities may develop at the biliary anastomosis secondary to compensatory hypertrophy of the liver allograft that leads to a sharp angulation of the bile duct (25). Such biliary strictures are difficult to treat by and the success rate after ERC alone is up-to $20 \%$. The NAS strictures are usually resistant to endoscopic management and ERC with BD of all stricture is not feasible and rapid stent clogging is frequently occurred while managing NAS biliary strictures. Such patients can be managed by a combined approach using ERC and PTC. In this series, 14 patients (17\%) were treated by combined approach without any reported recurrence. The stricture initially is accessed through PTC and the obstructed bile ducts are first decompressed to prevent acute iatrogenic cholangitis and the guide wire is then traversed across the stricture which is used to place stent by endoscopic route after performing dilatation of stricture. Use of the Kumpe (KMP) catheter in difficult NAS stricture is claimed to be more effective than a guide wire in terms of facilitating stent placement (26). In majority of the patients, a PTC drain is needed at first intervention to decompress the biliary system and reduce the biliary stasis. A combined ERC and PTC approach then undertaken to internalize the state that allows purely endoscopic approach for long-term therapy. Average ERC procedures required for group B patients $(E R C+P T C)$ were less $(3 \pm 2)$ than group $A$ patients (purely endoscopic approach). However, the time for resolution of stricture in combined approach was $1074 \pm 612$ days vs $706 \pm 526$ days for ERC patients which was significant $(p<0.05)$. The peculiar finding was the zero recurrence in patients with combined approach. The mortality rate directly related to biliary strictures was $24 \%$. The treatment could not be completed in these patients either due to late presentation of the patient with septic complications or loss of follow up. Post ERC complications such as bleeding, cholangitis, pancreatitis, biliary and duodenal perforation, and stent migration have been described and are cumulated with successive endoscopic procedures. The mortality directly related to complications occurring post-intervention was $6 \%$ in this series.

Recent developments in treating complex biliary strictures post-transplantation include new types of balloons and stents that will have significant role in 
improvement of management of biliary strictures. Recently, the role of self-expanding metallic stents (SEMS), which have significantly longer patency than plastic stents for palliation of malignant biliary obstruction, has been evaluated for treating the AS biliary strictures (27). However, because of factors such as smaller caliber bile ducts in LDLT, annular strictures preventing adequate stent anchorage, and migration of stent have led to disappointing results. In recently conducted randomized controlled trial involving SEMS vs plastic stents for treating post-LT biliary AS strictures, the resolution rates were comparable, but covered SEMS required less ERC procedures to achieve resolution (28).

\section{CONCLUSION}

In conclusion, biliary strictures in post-LDLT period remains a major source of morbidity and graft loss if not treated promptly. The endoscopic approach has become as the primary modality of treatment for biliary strictures. ERC and BD with multiple stent placement is a feasible and successful treatment modality that provides an increased resolution rate. A combined approach of ERC with PTC play complementary roles in patients with failed ERC or NAS biliary strictures not accessible by ERC.

\section{No conflicts of interest}

\section{No financial Funding from any source.}

\section{REFERENCES}

1. Ryu CH, Lee SK. Biliary Strictures after Liver Transplantation. GutLiver 2011; 5:133-142.

2. Verdonk RC, Buis $\mathrm{Cl}$, Porte RJ, et al. Anastomotic biliary strictures after liver transplantation: Causes and consequences. Liver Transpl 2006; 12: 726-735.

3. Freise CE, Gillespie BW, Koffron AJ, et al. A2ALL Study Group. Recipient morbidity after living and deceased donor liver transplantation: findings from the A2ALL Retrospective Cohort Study. Am J Transplant 2008; 8:2569-2579.

4. Guichelaar MM, Benson JT, Malinchoc M, Krom RA, Wiesner RH, Charlton MR. Risk factors for and clinical course of nonanastomotic biliary strictures after liver transplantation. Am J Transplant2003; 3: 885-890.

5. Poley JW, Lekkerkerker MN, Metselaar HJ, et al. Clinical outcome of progressive stenting in patients with anastomotic strictures after orthotopic liver transplantation. Endoscopy 2013; 45: 567-570.

6. Aparício DP da S, Otoch JP, Montero EF de S, et al. Endoscopic approach for management of biliary strictures in liver transplant recipients: A systematic review and meta-analysis. United European Gastroenterol J 2017; 5:827-845.

7. Jeng LB, Thorat A, Yang HR, et al. "Rooftop and Skeletonization Technique" of Hepatic Transection to Include or Exclude the Middle Hepatic Vein During Donor Hepatectomy in Living Donor Liver Transplantation: Solving the Middle Hepatic Vein Controversy -
Experience in 397 Sequential Live Donors. Med Sci Tech 2016; 57:6-15.

8. Jeng LB, Thorat A, Hsieh YW, et al. Experience of using everolimus in the early stage of living donor liver transplantation. Transplant Proc 2014; 46:744-748.

9. Samstein B, Smith AR, Freise CE, et al. Complications and their resolution in recipients of deceased and living donor liver transplants: findings from the A2ALL cohort study. Am J Transplant 2016; 16:594-602.

10. Liu CL, Fan ST, Lo CM, et al. Operative outcomes of adult-to-adult right lobe live donor liver transplantation: a comparative study with cadaveric whole-graft liver transplantation in a single center. Ann Surg 2006; 243:404-410.

11. Akamatsu N, Sugawara $Y$, Hashimoto D. Biliary reconstruction, its complications and management of biliary complications after adult liver transplantation: a systematic review of the incidence, risk factors and outcome. Transpl Int 2011; 24:379-392.

12. $L i P C$, Thorat $A$,Jeng $L B$, et al. Hepatic artery reconstruction in living donor liver transplantation using surgical loupes: Achieving low rate of hepatic arterial thrombosis in consecutive 741 recipients- Tips and tricks to overcome the poor hepatic arterial flow. Liver Transpl 2017; 23: 887-898.

13. Villa NA, Harrison ME. Management of biliary strictures after liver transplantation. Gastroenterol Hepatol2015; 11:316-328.

14. Seehofer D, Eurich D, Veltzke-Schlieker W, et al. Biliary complications after liver transplantation: old problems and new challenges. Am J Transplant 2013; 13:253-65.

15. Ostroff JW. Post-transplant biliary problems. Gastrointest Endosc Clin N Am 2001: 11:163-183.

16. Park JB, Kwon CH, Choi GS, et al. Prolonged cold ischemic time is a risk factor for biliary strictures in duct-to-duct biliary reconstruction in living donor liver transplantation. Transplantation 2008; 86:1536-42.

17. Seo JK, Ryu JK, Lee SH, et al. Endoscopic treatment for biliary stricture after adult living donor liver transplantation. Liver Transpl 2009; 15:369-80.

18. Guichelaar MM, Benson JT, Malinchoc M, et al. Risk factors for and clinical course of non-anastomotic biliary strictures after liver transplantation. Am J Transplant 2003; 3:885-90.

19. Thuluvath PJ, Pfau PR, Kimmey MB, et al. Biliary complications after liver transplantation: the role of endoscopy. Endoscopy 2005; 37:857-63.

20. Hussaini SH, Sheridan MB, Davies M. The predictive value of transabdominal ultrasonography in the diagnosis of biliary tract complications after orthotopic liver transplantation. Gut 1999; 45:900-903.

21. Morelli G, Fazel A, Judah J, et al. Rapid-sequence endoscopic management of posttransplant anastomotic biliary strictures. Gastrointest Endosc2008; 67:879-885.

22. Graziadei IW, Schwaighofer H, Koch R, et al. Long-term outcome of endoscopic treatment of biliary strictures after liver transplantation. Liver Transpl2006; 12:718- 725.

23. Thuluvath PJ, Pfau PR, Kimmey MB, et al. Biliary complications after liver transplantation: the role of endoscopy. Endoscopy 2005; 37:857-863.

24. Alazmi WM, Fogel EL, Watkins JL, et al. Recurrence rate of anastomotic biliary strictures in patients who have had previous successful endoscopic therapy for anastomotic narrowing after orthotopic liver transplantation. Endoscopy 2006; 38:571574.

25. Yoshimoto T, Yazumi S, Hisatsune $\mathrm{H}$, et al. Crane-neck deformity after right lobe living donor liver transplantation. Gastrointest Endosc 2006; 64:271.

26. Chang JH, Lee IS, Chun HJ, et al. Comparative study of rendezvous techniques in post-liver transplant biliary stricture. World $\mathrm{J}$ Gastroenterol 2012; 18:5957-5964.

27. Kao D, Zepeda-Gomez S, Tandon P, et al. Managing the post-liver transplantation anastomotic biliary stricture: Multiple plastic versus metal stents: A systematic review. Gastrointest Endosc 2013; 77: 679-691.

28. Martins FP, De Paulo GA, Contini MLC, et al. Metal versus plastic stents for anastomotic biliary strictures after liver transplantation: a randomized controlled trial. Gastrointest Endosc 2017; 87: 131.e1-131.e13. 